\title{
Single point incremental sheet forming investigated by in-process 3D digital image correlation
}

\author{
N. Decultot ${ }^{1,2}$, L. Robert ${ }^{1,2, a}$, V. Velay ${ }^{1,2}$, and G. Bernhart ${ }^{1,2}$ \\ 1 Université de Toulouse; INSA, UPS, Mines Albi, ISAE ; ICA (Institut Clément Ader); Campus \\ Jarlard, F-81013 Albi, France \\ 2 Ecole des Mines Albi, Campus Jarlard, F-81013 Albi, France
}

\begin{abstract}
Single Point Incremental Forming (SPIF) is a promising sheet metal forming process for prototyping and small batches, in which the blank is formed in a stepwise fashion by a displacement-controlled small-sized tool. Due to specific strain paths induced by the process and limited plastic zones in the contact region between the tool and the workpiece, forming diagrams and forming strategies are different from the classical stamping processes. One major limitation of SPIF is the lack of accuracy of the obtained final parts because of the poor knowledge of the state of stress during the process that requires a good description of the material models and a right choice of the process parameters. In this paper, the SPIF process is experimentally investigated by the mean of surface 3D digital image correlation during the forming of a AW-5086-H111 grade aluminium alloy. Development of strain fields encountered in incremental forming is reported and material formability is evaluated on several formed shapes, taking into account a wide range of straining conditions of this process.
\end{abstract}

\section{Introduction}

Single Point Incremental Forming (SPIF) is a sheet forming process that has gained interest recently in prototyping and small batch production. In this process, a sheet metal part (aluminium, steel) is formed in a stepwise fashion by a displacement-controlled hemispherical tool without the need for a supporting die [1]. This process enables the manufacturing of a desired shape by an incremental deformation in a small contacted region. Because of this slicing technique, complicated products can be fabricated by using a simple shaped punch driven by a numerically controlled milling machine or a robot with a non-dedicated die. As a result this process is characterised by a high flexibility which allows an interesting technology improvement to manufacture sheet metal parts in comparison with drawing process, and a reducing costs when prototypes or small batches have to be manufactured. Due to specific strain paths induced by the process and the fact that the plastic zone is strictly limited to the contact region between the tool and the work-piece, forming-limit curves and forming strategies are different from the classical deep drawing process [2]. The formability is generally characterized by the draw angle $\Phi$ (or wall angle $\alpha$ ), defined by the sine law $t_{f}=t_{0} \sin (\pi / 2-\Phi)=t_{0} \sin \alpha$ where $t_{0}$ and $t_{f}$ are the initial and final thicknesses of the part, respectively. The largest design draw angle $\Phi$ in the part must be less than $\Phi_{\max }$ which can be considered as a material parameter limit [1]. It is reported however that accuracy of the obtained shape due to springback effect [4], heterogeneous thickness strain distribution [3] and fabrication time are limitations of the process. Furthermore, simulations by Finite Element methods are time-consuming because of the incremental characteristics of the process [1]. However, these simulations need a good description of the process parameters, of the boundary conditions and of the material properties by the use of behaviour model representative of the

\footnotetext{
a e-mail: Laurent. Robert@mines-albi . fr
} 
incremental characteristics of the process, taking into account, for instance, the history of strain paths during the process. For studying these issues, a SPIF process pilot has been developed in the laboratory and instrumented with a force sensor to measure the resultant loading applied to the sheet metal part during the forming and with a multi-camera system to measure the shape variation of the sheet metal part and the 3D displacement/strain field of its surface by 3D-Digital Image Correlation (3DDIC) techniques. Quite recently, Vasilakos et al. [5] have also used the 3D-DIC technique to study the deformation phenomena of a two-angle pyramid sheet metal part during SPIF operation. They measured the out-of-plane displacement of only six points selected on the sheet metal and analysed the deviation (maximum displacement minus final displacement) during the forming. They concluded that the spring-back effect occurs immediately after the tool transit over the investigated material point and is only responsible for a minor fraction of the total geometrical error. However, they did not analysed directly strain fields.

The main objectives of this paper were to investigate, thanks to the high strain resolution and spatial resolution of the 3D-DIC technique, (i) the development of the strain fields encountered in incremental forming and (ii) the material formability in incremental forming, taking into account the wide range of straining conditions of the SPIF process. Therefore, different tests were designed: from a simple uniaxial tool path displacement over the blank with incremental penetration of the punch (Z-mouvement between successive loops), to crosses and pyramids, in order to cover a set of straining conditions ranging from uniaxial, pure uniaxial stretching, to fully biaxial stretching. In that conditions, some of the main SPIF parameters, typically the vertical increment step size $\Delta z$ and the tool diameter $D_{p}$ were investigated. Finally, forming diagrams were assessed.

In section 2, the design of the SPIF process pilot is first presented. Principle of the 3D-DIC is then recalled and chosen parameters are justified. In section 3, results of 3D-DIC full field measurements in term of strain fields, strain paths and forming diagrams for different tests are presented and discussed.

\section{Experimental equipment and method}

\subsection{The SPIF Process Pilot}

A SPIF process pilot has been developed in order to reproduce complex loading paths close to those induced in an industrial environment. It consists of a parallelepiped frame body. A properly designed blank holder was utilised to clamp the $500 \times 500 \mathrm{~mm}^{2}$ square blank on its top (see Figure 1). Below,
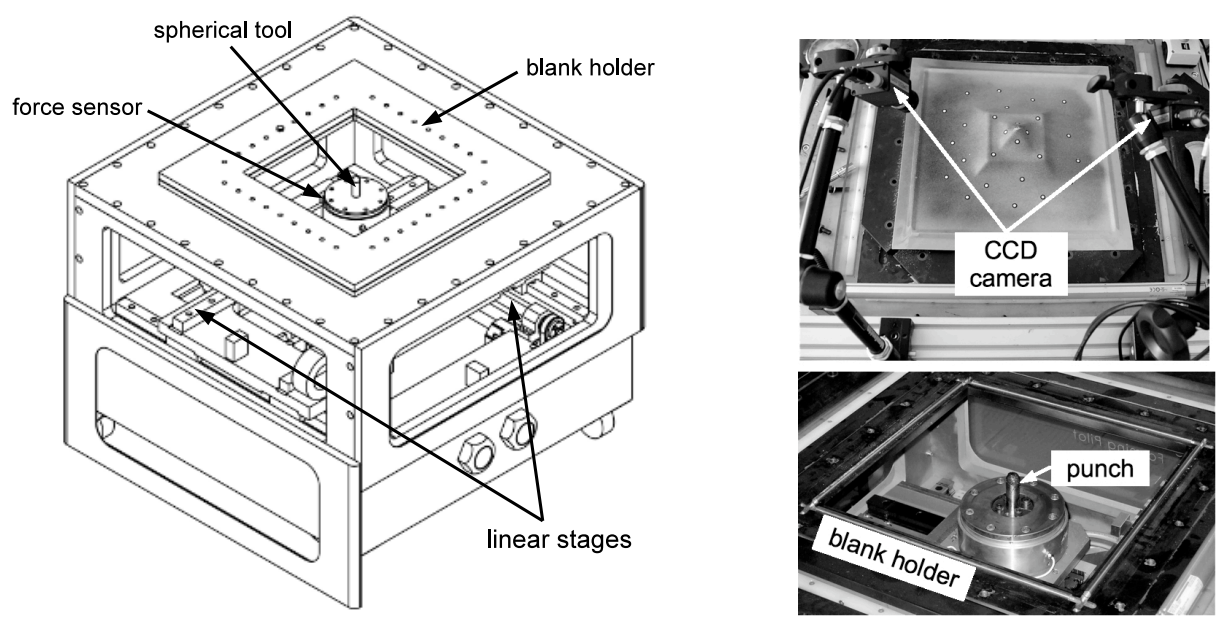

Fig. 1. The SPIF process pilot. CAD of the pilot (left), top view details (right).

a three axes motion of the tool (X, Y, Z axis directions) was provided by three linear stages with ball 
screw spindles. It allows on the one hand a vertical $(\mathrm{Z})$ tool displacement and a local stamping of the sheet and, on the other hand, a horizontal tool displacement in the frame of the sheet $(\mathrm{X}, \mathrm{Y})$ leading to the incremental forming of the piece. The tool path was specified by the pilot control with a computer connected to the later through a proper routine. The sheet deformation was performed with a 10 or 20 $\mathrm{mm}$ diameter hemispherical punch made of X38CrMoV5 tool steel. A $400 \times 400 \mathrm{~mm}^{2}$ work area can be studied in the current configuration of the machine and a $100 \mathrm{~mm}$ depth of draw can be reached. In order to form the piece, a bottom-top forming direction was considered. As a consequence, its design allows us the easy use of CCD cameras to perform in situ measurement of the deformation of the sheet by 3D-DIC (see section 2.2). Furthermore, a multi-axial sensor sets up the pilot that enables to monitor the three dimensional force measurement induced in the sheet by the punch displacement. The friction coefficient can be evaluated from this force measurement. For all the experiments, an EN AW-5086-H111 grade of aluminium alloy sheet of $1 \mathrm{~mm}$ thick was considered. A film of fluid grease was applied to the blank to reduce friction.

\subsection{D-Digital Image Correlation (3D-DIC)}

The 3D-DIC method is based on both digital image correlation (DIC) and computer stereo vision (utilisation of two cameras), and was developed at the end of the last century [6]. This technique uses a DIC algorithm to determine point correspondences between two images of an object to be measured, acquired from two different view points, by two rigidly bounded cameras. The correlation scores are computed by measuring the similarity of a fixed subset window in the first (left) image to a shifting subset window in the second (right) one. A first order subset shape function and a zero normalized sum of square difference (ZNSSD) correlation criterion are used. Sub-pixel correlation is performed using B-spline gray level interpolation. After determining the calibration parameters for each camera as well as the 3-D relative position/orientation of the two cameras (pinhole model and radial distortion of 1 rd order), the 3-D shape of the object can be reconstructed by triangulation from the image-points correspondences founded by DIC. To determine the 3-D displacement field, DIC is also used to determine image-points correspondences between the stereo pairs acquired before and after deformation (temporal tracking) [7]. The Hencky logarithmic surface strain field tensor is obtained from the displacement field by numerical differentiation. A complete description of the 3DDIC technique can be found in [8].

The stereo rig was composed of two 8 bit AVT Marlin F-145B2 CCD cameras allowing a $1392 \times 1040$ pixels resolution equipped with $25 \mathrm{~mm}$ lenses. Two synchronized images were taken at the frequency of $2 \mathrm{~Hz}$. The calibration and numerical image processing were carried out with the assistance of the Vic-3D commercial software [9]. As the DIC technique relies on a contrasting pattern on the surface of the test object, a randomly distributed white paint pattern was applied on the painted in black side of the sheet metal. A particular attention was paid to have a relatively fine speckle pattern size proportional to the region of interest, leading to a choice of a maximum $15 \times 15$ pixels $^{2}$ subset size (minimum $11 \times 11$ pixels $^{2}$ ). This allowed to increase the displacement spatial resolution in $\mathrm{mm}$ (subset size $\times$ magnification factor) because large strain gradients were present. Typical magnification factor of 8 $\mathrm{px} / \mathrm{mm}$ gave a $2 \times 2 \mathrm{~mm}^{2}$ displacement spatial resolution. The strain spatial resolution (optical gauge size) was also minimised and chosen equal to $2 \times 2 \mathrm{~mm}^{2}$. It has been shown in another study [10] that strain standard uncertainty can be estimated to $10^{-4}$ in similar experimental conditions.

\section{Results and discussion}

\subsection{Analyses of a simple uniaxial tool path}

In order to analyse the strain field encountered around the punch, a very simple tool path was first chosen. It consisted of a horizontal straight line of $100 \mathrm{~mm}$ length. After its mouvement along the line, the punch imposes the fixed depth to the blank. The increment step size was equal to $\Delta z=0.5$ $\mathrm{mm}$ and the tool diameter was $D_{p}=10 \mathrm{~mm}$. Along the straight line, a pure uniaxial stretching was 
observed. In order to have an area where almost pure biaxial stretching conditions must be achieved, a cross consisting of two perpendicular straight lines was finally formed [11]. A series of 171 pairs of images (171 steps) have been automatically taken before the ductile fracture occurs.

Figure 2(a) presents the 3D shape of the stamped cross measured by 3D-DIC for the step $\sharp 140$. Figure 2(b) reports the major strain field superimposed on the black and white painted speckle patterns

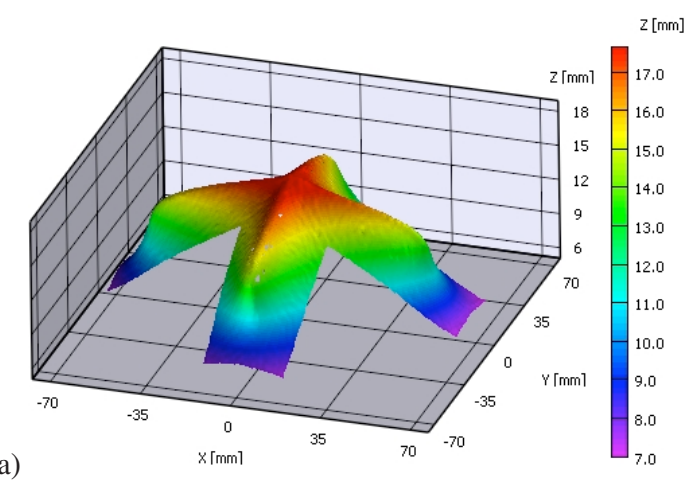

(b)

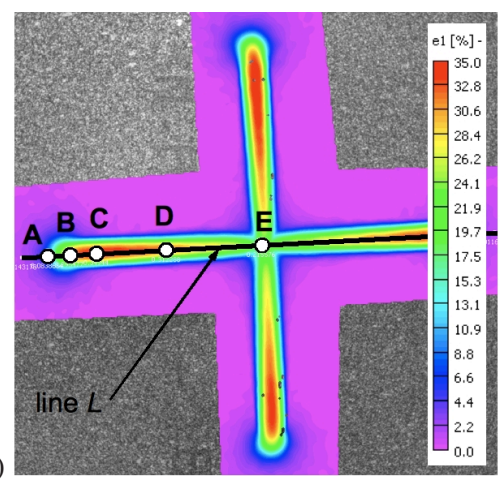

Fig. 2. (a) Shape of the formed part. (b) Maximum principal strain field and definitions of the line $L$ and areas $\mathbf{A}$ to $\mathbf{E}$. (Both at the step $\sharp 140$ of 171 stamping steps before fracture.)

of the blank, also for the step $\sharp 140$ which corresponds to a maximum major strain of about $35 \%$. It appeared that major strain was not constant along the straight line $L$ and maximum values were situated near area $\mathbf{C}$ (see Figure 2 for the definition of the line). An analysis of the straining evolution along line $L$ for several steps of the forming is presented in Figure 3 for both major and minor strains.
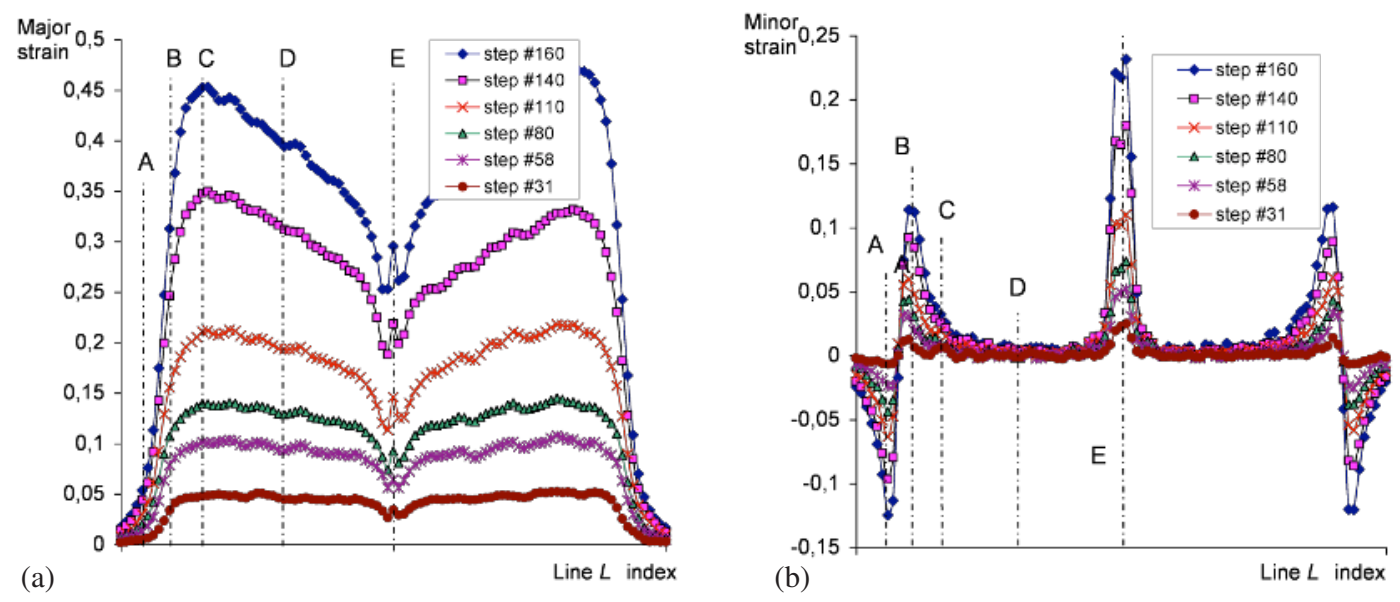

Fig. 3. Strain evolutions along line $L$ function of the depth of forming (see Figure 2 for the definition of line $L$ and areas). Left: major strain, right: minor strain. Only six image steps are presented, from step $\sharp 31$ to step $\sharp 160$.

Moreover, five areas (A to $\mathbf{E}$ ) along line $L$ defined in Figure 2 are reported on the plots in Figure 3. Area $\mathbf{A}$ corresponds to the minimum of minor strain, area $\mathbf{B}$ to the first maximum of minor strain, area $\mathbf{C}$ to the maximum of major strain, area $\mathbf{D}$ to the cancellation of minor strain, and area $\mathbf{E}$ to the maximum of minor strain which is roughly equal to the major strain. We show that, apart from some bending effects close to the stamped shape and the non-formed blank (area $\mathbf{A}$ and $\mathbf{B}$ ), the blank is mainly stretched by the local action of the punch: almost a pure uniaxial stretching was observed near the area $\mathbf{D}$, also observed in area $\mathbf{C}$, while almost a pure biaxial stretching was present in area $\mathbf{E}$. 
It was also observed that there was a large decrease of the major strain between $\mathbf{C}$ and $\mathbf{E}$ for almost no minor strain whereas the tool trajectory was a horizontal straight line. Moreover out-ofplane displacement $\mathrm{W}$ (which is quite equal to the $\mathrm{Z}$ value as the initial blank shape is flat) showed the inverse tendance: $\mathrm{W}$ increases continuously from areas $\mathbf{A}$ to $\mathbf{E}$, as shown in Figure 2, due to the other punch trajectory perpendicular to the later. This is more visible as the depth of punch penetration increases (steps $\sharp 110, \sharp 140$ and $\sharp 160$ ). As a probable consequence, the ductile tearing occurred near the area $\mathbf{C}$.

\subsection{Influence of increment step size and punch diameter}

The cross presented in Figure 2 have been formed considering two punch diameters $D_{p}=10$ and 20 $\mathrm{mm}$ and two increment step sizes $\Delta z=0.5$ and $2 \mathrm{~mm}$. Figure 4 shows strain paths of the five areas $\mathbf{A}, \mathbf{B}, \mathbf{C}, \mathbf{D}$ and $\mathbf{E}$ defined before on forming diagrams plots (major versus minor strain). Whatever
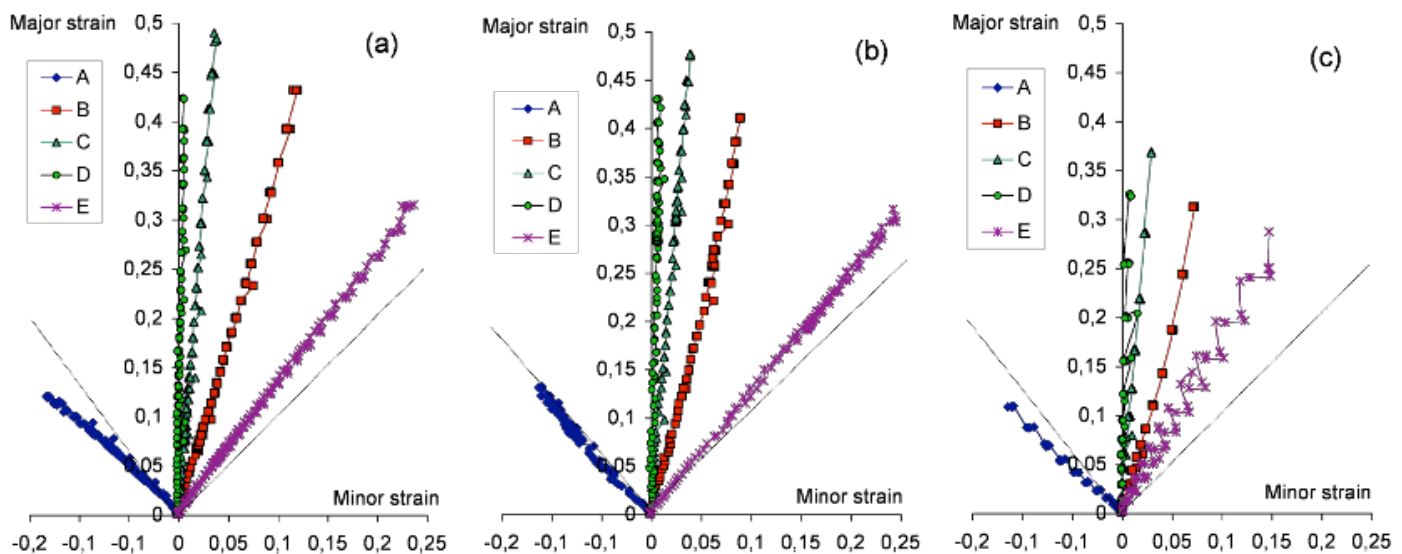

Fig. 4. Strain paths of the five areas (A, B, C, D and $\mathbf{E}$ on the stamped part, see Figure 2(b)) for two punch diameters $D_{p}$ and two increment step sizes $\Delta z$ : (a) $D_{p}=10 \mathrm{~mm}, \Delta z=0.5 \mathrm{~mm}$; (b) $D_{p}=20 \mathrm{~mm}, \Delta z=0.5 \mathrm{~mm}$; (c) $D_{p}=20 \mathrm{~mm}, \Delta z=2 \mathrm{~mm}$.

the punch diameter or increment step size, forming diagrams are very similar. Pure biaxial stretching is never totally reached (areas E), and the effect of the punch diameter (Figures 4(a) and 4(b)) is less significant than the effect of the increment step size (Figures 4(b) and4(c)). As reported before, the ductile tearing occurred near the area $\mathbf{C}$, permitting to detect the critical strain for quasi uniaxial stretching conditions. In the literature, most authors generally reported the maximum major strain at tearing for no minor strain in the Forming Limit Diagram (FLD), noted $F L D_{0}$ [1]. It is worth pointing out that hypothesis leading to FLD are not available in the case of SPIF, because through-thickness stresses are not negligible in that case of a small tool diameter. In our experiments, however, maximum major strain values before tearing (obtained between areas $\mathbf{C}$ and $\mathbf{D}$ ) were equal to $55 \%, 51 \%$ and $45 \%$ for respectively the following conditions $\left[D_{p}=10 / \Delta z=0.5\right],\left[D_{p}=20 / \Delta z=0.5\right]$ and $\left[D_{p}=20 / \Delta z=2\right]$ (in $\mathrm{mm}$ ). Values are slightly higher than those reported in Figure 4 because they are not exactly extracted at the physical point $\mathbf{C}$ or $\mathbf{D}$. Note also that in each case, minor strain do not exceed $4 \%$. To conclude, it is reported that sheet formability decreases with increasing increment step size and tool size [1], but fracture does not occur at the centre of the cross (biaxial stretching conditions) as reported in [11], but for almost uniaxial stretching conditions.

\subsection{Analyses of complex tool paths}

This section concerns the analysis of the deformation states that are able to obtain classically with the SPIF process by the stamping of pyramid-like parts. The first part was a small pyramid shell without 
rounding at the corners, except those due to the punch diameter (see Figure 5). The tool paths were square loops, each loop consisting of four straight lines. Dimensions of the first loop were $100 \times 100$ $\mathrm{mm}^{2}$. The depth increment step size was equal to $\Delta z=2 \mathrm{~mm}$ and the in-plane increment step size was equal to $\Delta x=\Delta y=2 \mathrm{~mm}$, as for a theoretical draw angle $\Phi$ of $45^{\circ}$, and the complete part was obtained after 25 loops. The tool diameter was $D_{p}=20 \mathrm{~mm}$.

(a)
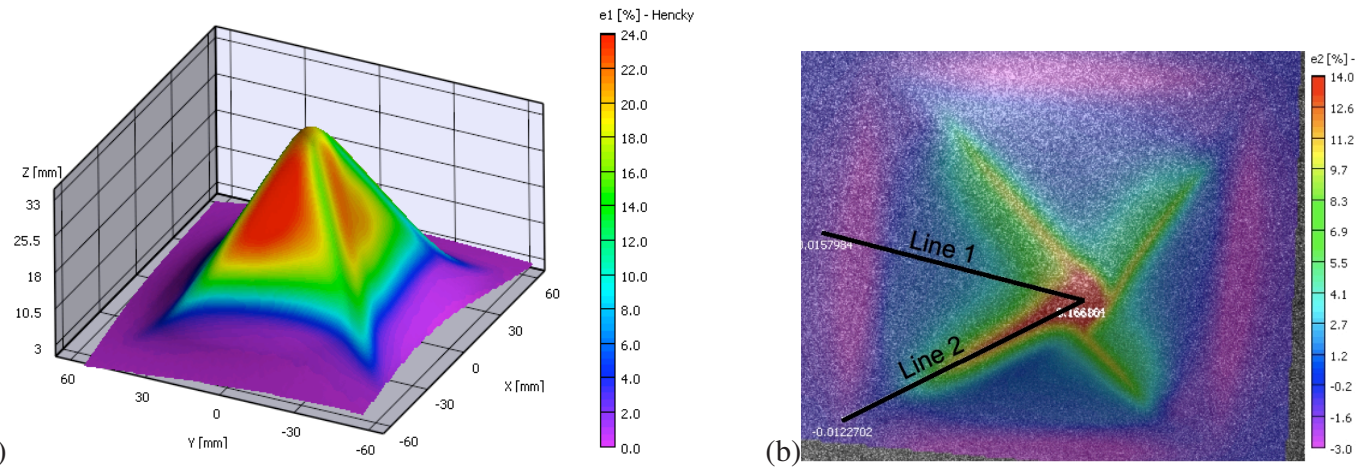

Fig. 5. Stamped small pyramid without rounding, shape measured by 3D-DIC for the last strain step. (a) Major strain field superimposed on the 3D shape. (b) Minor strain field and definition of lines 1 and 2 (planar view).

The second part was a large truncated pyramid shell with rounding at the corners (see Figure 6). The tool paths were square loops with rounding at the corners. The rounding radius was kept constant and equal to $50 \mathrm{~mm}$ while the dimensions of the first loop are $240 \times 240 \mathrm{~mm}^{2}$. The depth increment step size was varying for the first five loops respectively as $\Delta z=\{10 ; 8 ; 6 ; 4 ; 2\} \mathrm{mm}$ and then was kept constant and equal to $\Delta z=2 \mathrm{~mm}$. The in-plane increment step size $\Delta x=\Delta y$ was always equal to $\Delta z$, as for a theoretical draw angle $\Phi$ of $45^{\circ}$. The complete part was obtained after 25 loops so that the height of the formed part was $10+8+6+4+2 \times 21=70 \mathrm{~mm}$, implying that the last tool path was exactly a circle of $50 \mathrm{~mm}$ diameter as it can be seen on the top of the truncated pyramid in Figure 6.

(a)

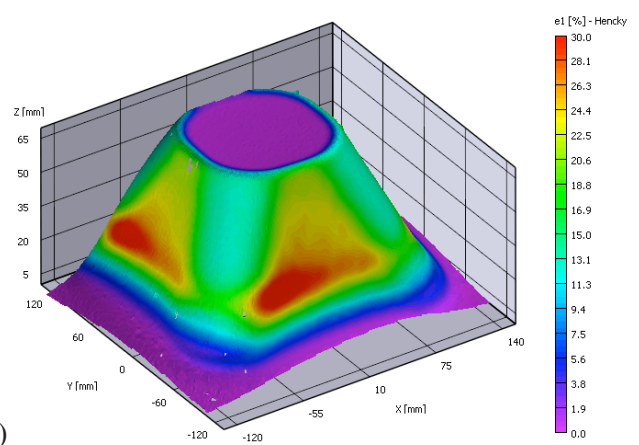

(b)

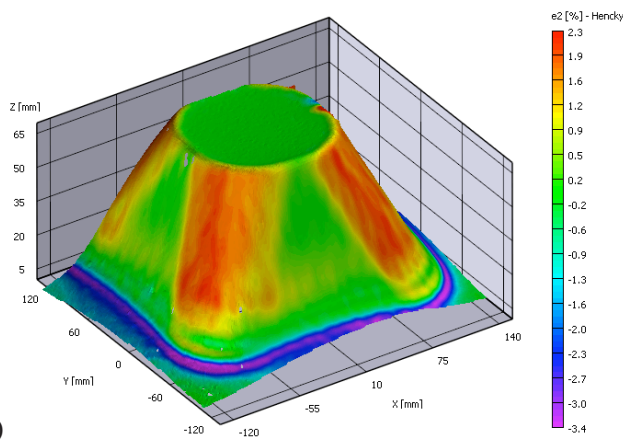

Fig. 6. Stamped large truncated pyramid with rounding at the corners, shape measured by $3 \mathrm{D}$-DIC for the last strain step. (a) Major strain field superimposed on the 3D shape. (b) Minor strain field.

On the one hand, it is important to note that thanks to the high resolution of the 3D-DIC method, the influence of wear between the punch and the blank was clearly observed. In these experiments, clockwise punch displacement for each loop created a distortion because of the rotation of the stamped part locally pulled by the punch. These distortions can clearly be seen in Figure 5 and result in asymetric strain fields on both flat faces of the pyramid. Note also that due to the ratio between punch diameter 
and part size, this effect is widely reduced for the large truncated pyramid but can also be observed in Figure 6.

On the other hand, it is also possible to analyse the evolution of the strain amplitude during the incremental forming of the pyramid. Figure 7(a) presents the major strain along line 6 (defined in Figure 7(b)) for all the 25 incremental forming loops of the truncated pyramid. As expected, the first

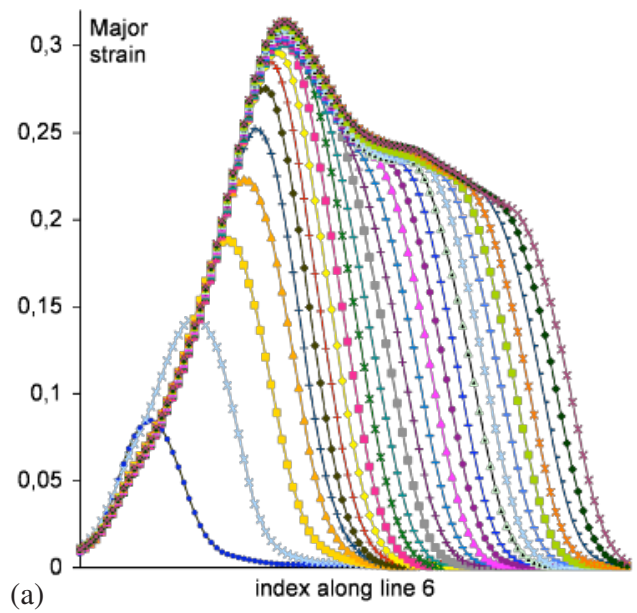

Fig. 7. Large truncated pyramid. (a) Evolution of the major strain along line 6 for each of the 25 incremental loops. (b) Definition of the lines in the quarter of the pyramid (minor strain map).

five loops for which $\Delta z$ was respectively equal to $10 ; 8 ; 6 ; 4$ and $2 \mathrm{~mm}$ gave the larger strain increment amplitude, respectively $8.43 ; 5.81 ; 4.46 ; 3.56$ and $2.9 \%$. The variation between the increment step size and the strain increment is non linear, probably due to structural and spring-back effects. After about the tenth loop, surprisingly, the major strain increment amplitude highly decreases whereas the punch continues to formed with a constant depth increment step size of $2 \mathrm{~mm}$. As a consequence, the major strain decreases globally along line 6 from $31 \%$ to $21 \%$, leading to the heterogeneous major strain field along $Z$ on the flat faces observed in Figure 6(a). This trend was not observed for the small pyramid.

Finally, from the strain fields measured by 3D-DIC, the forming diagrams for both pyramid stamped parts can be plotted (see Figure 8). As a first remark, large biaxial stretching was obtained for the small pyramid because there was no rounding at the corners. Indeed, if the corner radius is simply equal to the one of the hemispherical head of the punch, nearly biaxial stretching conditions are achieved in the material. Consequently, uniaxial strain field was almost homogeneous excepted along the corners. This is illustrated by the analysis of the strain along two specific lines: line 1 (maximum major strain area) and line 2 (maximum minor strain area), superimposed on the forming diagram of Figure 8(a), for several image steps. As expected we observe that the blank undergoes a nearly pure biaxial stretching along line 2 (maximum 17\% of minor strain is achieved for $20 \%$ of major strain) and a nearly pure uniaxial stretching along line 1.

Secondly, it appeared also that a small but positive and homogeneous minor strain field was obtained at the corners of the large truncated pyramid. Minor strain along the first five lines in Figure 8 (b) reaches about only $2 \%$ (see Figure 7(b) for the definition of the lines), comparatively to the $17 \%$ in Figure 8(a), for almost a similar level of major strain $(25 \%)$. So by decreasing the corner radius, from an infinite value (equivalent to a quasi straight line punch displacement $i e$ pure uniaxial stretching conditions) to a radius equal to the one of the punch (equivalent to a simple spherical drawing, ie pure biaxial stretching conditions), for instance by forming a cone, it is thus possible to fully scan the positive area of the forming diagram, that is to say the ratio between principal strains. The level of principal strain values is managed by the wall angle $\alpha$ via the sine law, which will be studied in the forthcoming work. 

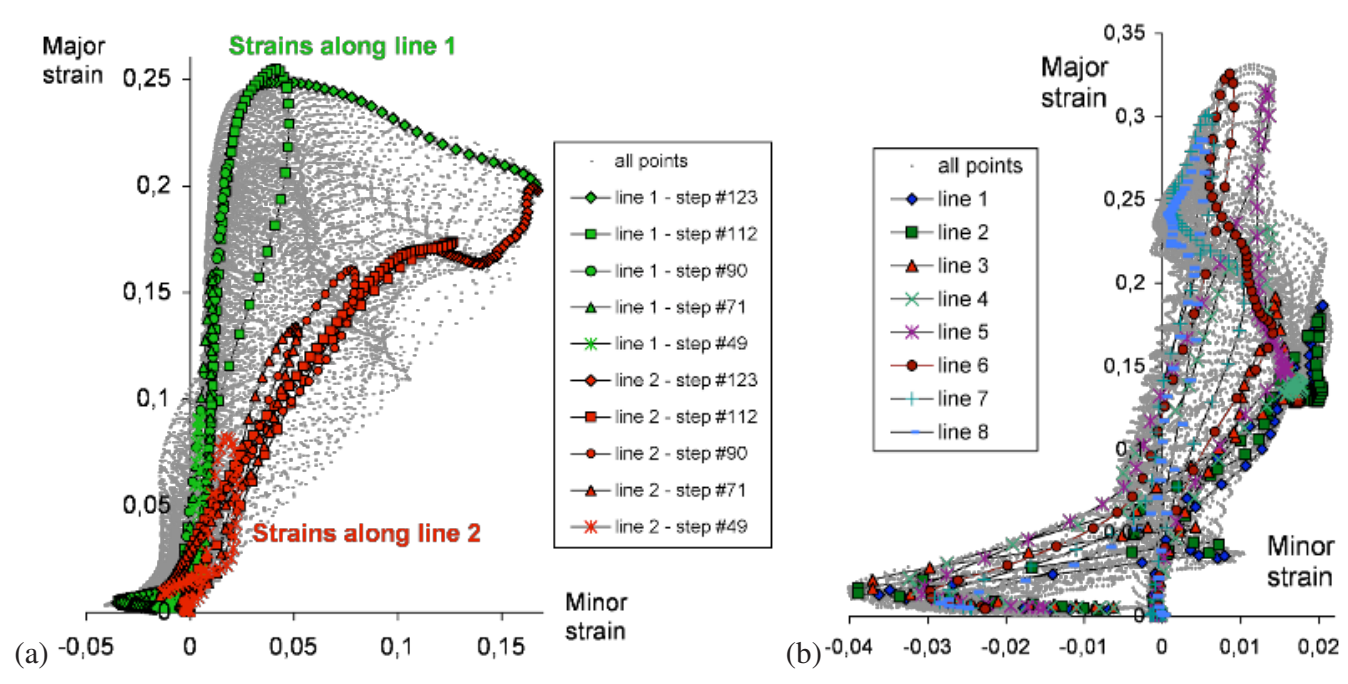

Fig. 8. Forming diagrams for both pyramid-like stamped parts. (a) Small pyramid without rounding. In bold symbols are represented the strain paths along lines 1 and 2 defined in Figure 5(b) for several image steps from step $\sharp 49$ to last step $\sharp 123$. (b) Large truncated pyramid with $50 \mathrm{~mm}$ radius roundings. In bold symbols are represented the strain paths along the 8 lines defined in Figure 7(a) for the last forming step.

\section{Conclusion}

We have investigated in detail, thanks to the high strain resolution and spatial resolution of the 3D-DIC technique, the development of strain fields encountered in incremental forming of a AW-5086-H111 grade of aluminium alloy. The material formability in incremental forming have been evaluated, taking into account the large range of straining conditions of this process. The analysis of the formed cross has shown that fracture occurs in the uniaxial stretching domain, and $F L D_{0}$ have been set to $55 \%$ for this material. It is also experimentally reported that sheet formability decreases with increasing increment step size and tool size. Pyramid experiments have shown that straight line punch displacement involves pure uniaxial stretching. When the punch displacement is curvilinear, a small level of minor strain for a given major strain can be measured that becomes very important for a bending radius which tends toward the radius of the hemispherical tool (pure biaxial stretching conditions).

\section{References}

1. Jeswiet, J., Micari, F., Hirt, G., Bramley, A., Duflou, J., Allwood, J., CIRP Annals 54, (2005) 623

2. Jeswiet J., Young D., Proc. IMechE Part B: J. Eng. Manufacture 219, (2005) 359

3. Kim, T. J. and Yang, D. Y., Int. J. Mech. Sci. 42, (2000) 1271

4. Micari F., Ambrogio G., and Filice L. , J. Mat. Proc. Tech. 191, (2007) 390

5. Vasilakos I., Gu J., Belkassem B., Sol H., Verbert J., and Duflou J. R., Key Eng. Mat., 410411(2009) 401

6. J. Helm, S. McNeill and M. Sutton, Opt. Eng. 35, (1996) 1911

7. D. Garcia, J.J. Orteu, L. Penazzi, J. Mat. Proc. Tech. 125, (2002) 736

8. M. A. Sutton, J.-J. Orteu and H. Schreier, Image Correlation for Deformation and Shape Measurements: basic concepts, theory and applications (Springer, 2009) 364p.

9. Vic-3D software, Correlated Solutions Inc., (2010) http://www.correlatedsolutions.com/

10. L. Robert, F. Nazaret, T. Cutard and J.-J. Orteu, Exp. Mech. 47, (2007) 761

11. L. Filice, L. Fratini, F. Micari, CIRP Annals 51, (2002) 